$\underline{\text { Review Article }}$

\title{
POWDER SOLUTION TECHNOLOGY REVIEW
}

\author{
SHAVETA SHARMA ${ }^{1}$, VIMAL ARORA* \\ ${ }^{1}$ Chandigarh College of Pharmacy, CGC Campus, Landran, Mohali, Punjab (India) 140307, "University Institute of Pharma Sciences, \\ Chandigarh University, NH-95, Chandigarh Ludhiana Highway, Mohali, Punjab (India) 140413 \\ "Email: vimalarora2022@gmail.com
}

Received: 02 May 2021, Revised and Accepted: 27 Jun 2021

\begin{abstract}
Bioavailability and Solubility are the challenges for the formulation of highly lipophilic drugs. Oral routes of administration is one of the acceptable route due to improved patient compliance and convenience. Regularly newly advanced drug candidates are lipophilic, BCS Class II and IV drugs. Among various methods to improve the solubility of these drugs, liquid-solid technology or Powdered solution technology change the liquid drug into non-sticky, dry free-flowing, rapid release powder. This technique involves mixing of insoluble drug with nonvolatile solvent, admixing of drugloaded excipients change into loose powder. This technique enhances major challenges like bioavailability with low production cost and a simple manufacturing process.
\end{abstract}

Keywords: Liquisolid technology, Bioavailability, Dissolution enhancement, Lipophyllic drugs, Solubility

(c) 2021 The Authors. Published by Innovare Academic Sciences Pvt Ltd. This is an open access article under the CC BY license (https://creativecommons.org/licenses/by/4.0/) DOI: https://dx.doi.org/10.22159/ijcpr.2021v13i4.42739 Journal homepage: https://innovareacademics.in/journals/index.php/ijcpr

\section{INTRODUCTION}

Mostly newly developed drugs are Lipophyllic which generally faces challenges like solubility and bioavailability. Numerous methods like saltformation, [1] cosolvancy, [2] complexation, [3 ]micronization, [4] melts onocrystallization, $[5,6]$ lyophilization, [7], solubilization bysurfactants [8] solid solution [9], drug solution incorporation in soft gelatin capsule [10] liposomes, nanoparticles, SEEDS, [11] imroves the dissolution of the drugs of low solubility These techniques have some limitations such as hygroscopicity, creating solubilityproblem [12,13]. The word liquid medicine means oily liquid drugsolution or suspensio $\mathrm{n}$ held as liquid vehicles in appropriate nonvolatile solvent systems.

"Liquisolid Tablets" or "Liquisolid Compacts" not involve drying and evaporation [14]. In tabletted and capsulated form drug is embedded in liquid [15] so this technique is known as "PowderSolution Technology". Greater surface area and adsorption of carrier materialto adsorb adequate space in liqyidmedicine. Usually Carrier adsorb liquid on its surface with a very large surface area and coating material forms the layer on carrier particles represented [16] [fig. 1.

\section{Classification}

A. Liquid medication within the systems: Powdered drug solutions and suspensions have the concept of changing them into liquisolid systems with its formulation. Liquid drug is circulate all over the final product.

B. Formulation technique:. Liquisolid compacts-Immediate sustained-release tablets or capsules whereas the microsystemsLiquid medication is integrated towards excipients and give freerunning powder for encapsulation [17].

\section{Mechanism}

\section{Surface area of drug incresed}

Molecular dispersed state-Region of the product which is accessible to discharge is beyond rather product molecules in the strictly constricted state [18].

\section{Poarity enhanced}

Liquid vehicle scatter in a single liquisolid particle jointly with the drug molecules is acceptable to improve the water solubility of the drug at the solid/liquid intersection among distinct liquisolid primary particle and the release medium [19].

\section{Improved wetting properties}

Wet ability is an indication of calculating contact angles and water rising times [20]. It will increase the drug release of many poorly soluble drugs.

\section{Theory}

Compressible liquid retention potential ( $\psi$-value): Uttermost of liquid that a powder can maintain within its bulk $(\mathrm{w} / \mathrm{w})$ while keeping reasonable compatibility, produces cylindrical compacts of adequate crushing strengths liquid loading capacity of powders: A mathematical approach is used to enumerate the amount of carrier and coating materials for the management of Liquisolid systems [21, $22,27,28]$. With the help of angle of repose, flowability can be determined.

Liquid load factor (Lf) Refers to scale among the weights of liquid formulation (W) and the carrier material (Q): $W / Q$,

$\mathrm{R}$ means equation among the weights of the carrier $(\mathrm{Q})$ and coating (q) material Q/q [23] the techniques for increasing solubility are enclosed in fig. 1 .

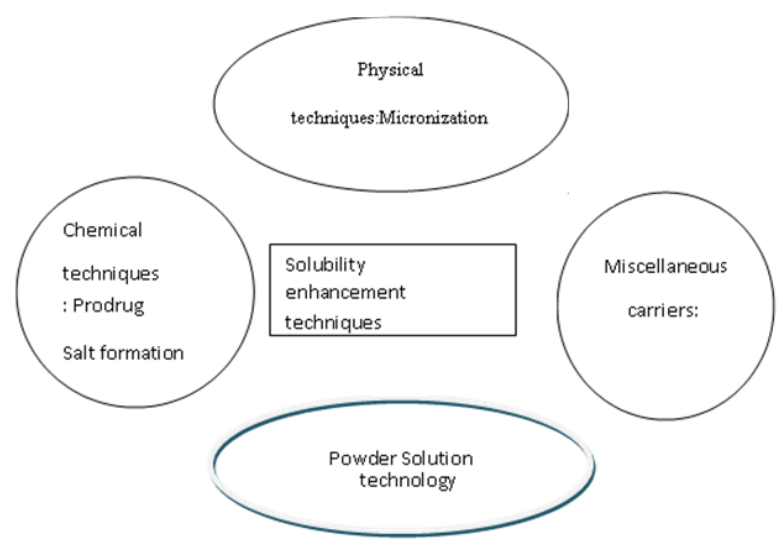

Fig. 1: Techniques for increasing solubility

Preparation of Liquisolid powder which can be incorporated in capsules and punched into tablets are enclosed in given fig. 2

\section{Formulation components}

Components such as Nonvolatile solvent, Carrier and Coating materials, Disintegrating agents and lubricants are used in the 
formulation of Liquisolid compact non-volatile solvent: Non toxic, great boiling point,good solubilization power and also work as binder. Eg: Polyethylene glycol 200 and 400, Glycerine. Polarity and lipophilicity are important parameters on drug release profiles [21] It is a good binder in low concentration for compactness of liquisolid tablets. Lower tablet weight is achieved with more solubility of drug in the solvent. The fragment of the molecularly diffused drug will confirms the enhancement of the dissolution rate $[24,25]$.

Carrier Materials: Compression-enhancing, relatively large, porous surface and high liquid adsorption function. eg. Cellulose, starch and glucose. Coating material influences the carrier material like polarity and viscosity [26]. MCC PH 101 is a worthy carrier amid all the grades of MCC (i.e., PH 101, 102, and 200) in liquisolid system concerning flowability, compressibility, and dissolution profile [27].

Coating Material: It will make a film that surrounds the carrier material which stops the gathering of particles and also decrease the inter-particulate friction. By adsorbing an excess liquid it enhances flowability and gives a dry-looking appearance [28]. e. g. Various grades of colloidal silica

Disintegrating Agents: They splits the solid into little particles and the incorporation of super disintegrants is encouraged for solubility enhancement studies. eg sodium starch glycolate Various excipients used in the preparation of Liquisolid powder are enclosed in table 1

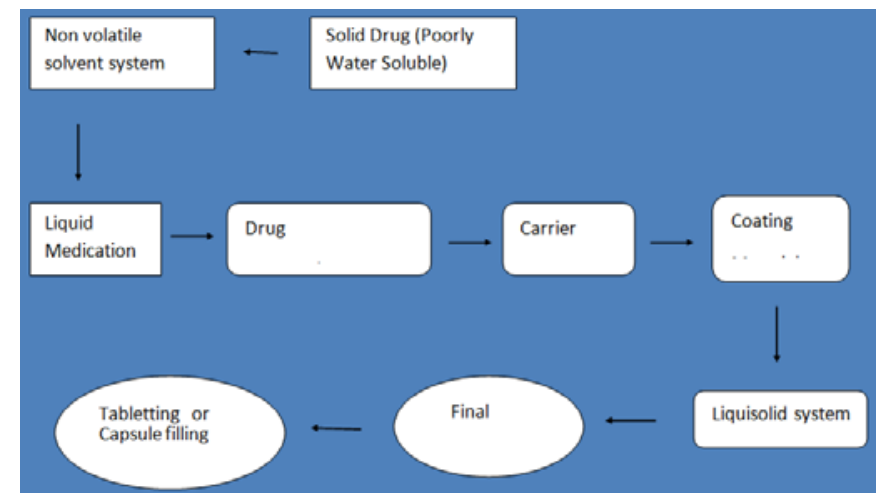

Fig. 2: General steps to prepare liquisolid formulation

Table 1: List of major excipient used to prepare suitable liquisolid formulation

\begin{tabular}{lllll}
\hline S. No. & Nonvolatile solvent & Carrier materials & Coating materials & Disintegrants \\
\hline 1. & Glycerine & MCC Avicel pH 101,102,105,200 & Colloidal Silica (Aerosil200) & $\begin{array}{l}\text { Polyvinylpyrrolidine } \\
\text { Sodium Starch Glycolate (SSG) } \\
\text { (Explotab) }\end{array}$ \\
& Propylene Glycol(PG) & $\begin{array}{l}\text { Fujicalin (Dibasic Calcium } \\
\text { Phosphate) }\end{array}$ & Fused Silica (Cab-o-Sil M5) & Cross Sodium carboxymethyl cellullose \\
(Croscarmellose Sodium) & Pregelatized Starch \\
& Polyethlene Glycol PEG & $\begin{array}{l}\text { Neusilin (Magnesium } \\
\text { aluminometasilicate) }\end{array}$ & Syloid 244FP & Colloidal Silicon dioxide \\
5 & $200,300,400,600$ & & \\
6 & Polysorbate $20,40,60,80$ & Eudragit RL & & \\
7 & Tween 80 & Eudragit RS & & \\
8 & Clive Oil & HPMC-E15 & & \\
9 & Soyabean oil & Xanthum Gum & \\
\hline
\end{tabular}

\section{Preformulation studies}

Solubility study of drug in non-volatile sovent: Pure drug liquefy in distinct non-volatile solvents and extreme, pure drug were joined shift to a rotatory shaker at $25^{\circ} \mathrm{C}$ under constant vibration for $48 \mathrm{~h}$ $0.45 \mu \mathrm{m}$ Millipore filter used for refining the saturated solution then analyzed [29].

Determination of angle of slide: In polished metal plates, liquid/powder admixtures were settled and plate tilted. The inclination set up in middle of the plate and horizontal surface (h) [30].

Determination of Flowable Liquid Retention Potential ( $\Phi$ value)

$\Phi-$ value $=$ weight of liquid $/$ weight of solid ....

Liquid Load Factor $\left(\mathrm{L}_{\mathrm{f}}\right)$

$\mathrm{L}_{\mathrm{f}}=\mathrm{W} / \mathrm{Q} \ldots \ldots \ldots \ldots \ldots \mathrm{W}=$ weight of liquid medication, $\mathrm{Q}=$ weight of carrier material

$\mathrm{R}=\mathrm{Q} / \mathrm{q} \mathrm{R}$ (ratio of the weight of carrier and coating material present in the formulation) [31]

\section{Formulation Steps to prepare liquisolid compact}

This preparation is mainly for Lipophyllic drugs. Drug liquefy in non volatile solvent to make drug solution. Mixing should be such that one rotation per second till one minute. Liquid medication extent as a uniform layer on the surface for 5 min to allow the drug solution to be absorbed inside the powder particles. Carrier and coating material is incorporated in the ratio of 20:1 to this mixture and blended. Final formulation can compress into tablet or filled into a capsule.

\section{Characterization of liquisolid system}

The evaluation of liquisolid powder like bulk density, tapped density, \% Compressibility Index and Haunsers ratio which exhibits the powder with low interparticle is required. In Differential Scanning Calorimetry drug ( 3 to $5 \mathrm{mg}$ ) evacuated in aluminium pans bares the temperature range of 30 to $300{ }^{\circ} \mathrm{C}$. Thermal behavior is examined and in X-Ray Diffraction Studies the machine usually serves at an angle 5 to $70^{\circ}$ and counting rate of $0.45 /$ step, use a $30 \mathrm{~mA}$ current and a copper target of voltage $40 \mathrm{KV}$. Peak pattern explains change of crystalline state to amorphous. Scanning Electron Microscopy estimates the surface behaviour of the drug. Due to the dissolving nature of the drug, molecular forms can get loss. Fourier Transform Infrared spectroscopy gives information that there is compatibility among drugs and excipients the absence of chemical interaction shown by the peaks. Post compression parameters include weight variation, Friability and Disintegration test In vitro Drug Release Studies which involves USP dissolution apparatus type 
II,900 $\mathrm{ml} 0.1 \mathrm{~N} \mathrm{HCl}$ at constant temperature of $37^{\circ} \mathrm{C} \pm 2$ and at speed of 50 to $200 \mathrm{rmp}$ [32 ]. In vivo Evaluation of Liqisolid Tablets: Relative bioavailability and Area under plasma concentration display mobile differences among the liquisolid compact and a commercial tablet [33]. Outcomes of Liquisolid technology are enclosed in given table 2

Table 2: Workout and results of liquisolid technology

\begin{tabular}{|c|c|c|}
\hline $\begin{array}{l}\text { S. } \\
\text { No. }\end{array}$ & $\begin{array}{l}\text { LST concept } \\
\text { (Year wise) }\end{array}$ & Investigation reported or significance [34] \\
\hline 1 & 2007 & $\begin{array}{l}\text { Initiation of concept: Liquisolid tabletslike Prednisolone, methylclothiazide, Hydrochlorothiazide and piroxicamboost up the } \\
\text { dissolution profiles as related to Direct compressible tablet. }\end{array}$ \\
\hline 2 & 2008 & $\begin{array}{l}\text { Tablete prepared by Liquisolid technology of Carbamazepine, Famotidine, Propranolol HCL and Bromhexine prove that } \\
\text { drug release not only depend on solubility in non volatile solvent but also depend on surface of carrier material, } \\
\text { physiochemical properties }\end{array}$ \\
\hline 3 & 2009 & $\begin{array}{l}\text { Numerous grades of MCC, Propylene Glycol, Silica used in Indomethacin Liquisolid tablets, dissolution was improved by } \\
\text { MCC. }\end{array}$ \\
\hline 4. & 2010 & $\begin{array}{l}\text { Drug release rate, dissolution profile and Bioavailability of Liquisolid compact is higher as compared to DCT. Significant } \\
\text { enhancement in Aceclofenac and Rofecoxib Liquisolid tablet as compared to commercial product. }\end{array}$ \\
\hline 5. & 2011 & $\begin{array}{l}\text { Fujicalin (Dibasic Calcium Phosphte) and Neusilin (Magnesium alumino metasilicate) are more effective carrier materials } \\
\text { than Avicel(Microcryatalline cellulose) compared. Dissolution rate and bioavailability of Glipizide, Indomethacin, } \\
\text { Lansoprazole, is enhanced and dissolution profile of simvastatin Liquisolid tablet show } 90 \% \text { release with } 45 \text { min. }\end{array}$ \\
\hline 6 & 2012 & $\begin{array}{l}\text { From all carrier material used MCC shows higher dissolution among all developed liquisolid tablet such as Nimesulide, } \\
\text { Loratidine, Ketoprofen and Griseofulvin and also proves PEG } 400 \text { is better than PG }\end{array}$ \\
\hline 7 & 2013 & $\begin{array}{l}\text { Amlodipine, Candesartancilextil, Mefanamicacid, Rosuvastatin, SpirinolactoneLiquisolid Tablets showed better release } \\
\text { retardation Trimetazidine dihydrochloride sustained releae tablet by using Liquisolid technology proves show that } \\
\text { polysorbate } 80 \text { also used ad liquid vehicle in sustaining the release of drug from liquisolid matrices [35] }\end{array}$ \\
\hline 8 & 2014 & $\begin{array}{l}\text {,Physicochemical characteristic among all Clonazepam, Candesartan,Lamotrigine and nateglimide Liquisolid Tabletes. } \\
\text { Solubility and dissolution rate of piroxicam is increased by Span 20, Tween 80, PEG 400,Labrosol.[36] }\end{array}$ \\
\hline 9 & 2015 & Hydrochlorthiazide and Domperidone maleate LSC showed improvement in dissolution rate and solubility \\
\hline 10 & 2016 & $\begin{array}{l}\text { Liquisolid pellets of Felodipine (101) and Curcumin loaded Liquisolid systems using different vechicles in different } \\
\text { concentration enhances the drug dissolution }\end{array}$ \\
\hline 11 & 2017 & LSC of Clinidipine in Tween 80 boost up the dissolution rate than marketed tablet based upon solubility.[37] \\
\hline 12 & 2018 & LST enhance solubility of BCS class II and IV (Loperamide, Furosemide) as compared to pure drug[38] \\
\hline 13 & 2019 & $\begin{array}{l}\text { Crystalline state of drug is changed to amorphous statein Curcumin Liquisolid tablets exhibited improvement in dissolution } \\
\text { rates as well as apparent solubility was obtained [39] }\end{array}$ \\
\hline 14 & 2020 & $\begin{array}{l}\text { Proves difference between Liquisolid pellet and liquipellet Liquisolidpellet uses liquisolid system and Liqui-Pellet uses liqui- } \\
\text { mass system [40] }\end{array}$ \\
\hline
\end{tabular}

\section{Liquisolid system for controlled drug delivery}

Therapeutic concentration of drug is maintained in the blood throughout the dosing interval with the help of this controlled drug delivery, this technique has the capacity to be optimized for the reduction of drug dissolution rate and thereby production of sustained release systems. Sustained release systems can be obtained by using hydrophobic carriers. Encapsulation of drug particles by hydrophobic polymers are more efficient than hydrophyllicpolymers. Polymer network surrounds the drug as leaching is not possible so easy to sutain the release of drug from Liquisolid matrices [41]. Efficacy, Patient Compliance and safety in formation of sustained-release oral dosage form.

\section{Advantages}

Low production cost. The Bioavailability of BCS class II and IV drugs can be improved manufacturing cost of formulations is lowest as compared to soft gelatin capsules drug release modification is achieved with the help of suitable ingredients. They are very ductile. Improves the drug release by using certain hydrophobic carriers and surface-active agents thus enhances the dissolution profile. The manufacturing capability can be increased. The extent of absorption is better than conventional tablets

\section{Limitations}

Inadequate hardness achieved if the acceptable compression is not achieved This results in a decreased tablet size by the substances with greater absorption rate

\section{Applications}

This system act as a weapon to increase drug dissolution: Felodipine Liquisolid pellet can be prepared with the help of this technique, Hydrochlorothiazide Liquisolid tablets by in vivo studies proves significant bioavailability rather commercial oral dosage forms, Sustain drug release: Venlafaxine Hydrochloride Liquisolid tablet having larger retardation effect contrast to DCT.
Minimize the influence of $\mathrm{pH}$ variation on drug release: Minimizes the influence of $\mathrm{pH}$ in release of Loratidine Liquisolid tablet. It increases solubility and dissolution rate in drugs Sustained-release tablets can be formulated with hydrophobic

\section{Current reports}

Liqui-mass system is a fundamental difference between liquisolid technology and liquid-Pellet technology (also referred to as LiquiMasstechnology). There is a strategy to increase the ritonavir dissolution rate.[42] Liqui pellet (Liqui mass System)the emerging next-generation oral dosage form which stems from liquisolid concept incombination with pelletisation technology using deionized water granulating liquid,29\% Non-volatile organic solvent, Aerosil 300 as coating material, liquid load factor 1 by oven drying method. Liquisolid technology (Liquisolid system)applied to pellets: evaluation of the feasibility and dissolution performanusing felodipine as a model drug using copovidone in water (1\%) granulating liquid, 5\% Non-volatile organic solvent, crospovidone (also disintegrant) as coating material, liquid load factor 0.1 by Fluid bed dryer $[43,44]$.

\section{CONCLUSION}

This present review shows that numerous techniques are used to increase solubility and bioavailbilty of highly lipophllic drugs among all liquisolid technique act as a favourable technique for crushing these challenges. These tasks are enhanced as rise in wetting properties and surface area of the drug usable for dissolution medium. Drug release. can be modified by suitable disintegrating agents, carrier and coating materials,. It has good production capability and formulations are of lower cost. Patient compliance in oral route grabby the technology will be high. This study proves that Liquisolid technology can be used effectively for the poorly soluble drugs and this technique is truly favorable for BCS class II and class IV drugs

\section{ACKNOWLEDGEMENT}

The authors are grateful to the directors of Chandigarh group of college and Chandigarh University for providing an opportunity to write a review in this topic. 


\section{FUNDING}

Nil

\section{AUTHORS CONTRIBUTIONS}

All the authors have contributed equally.

\section{CONFLICT OF INTERESTS}

Declared none

\section{REFERENCES}

1. Parikh RK, Mansuri NS, Gohel MC, Sonwala MM. Dissolution enhancement of nimesulide using complexation and salt formation techniques. Indian Drugs 2005;42:149-54.

2. Nayak AK, Panigrahi PP. Solubility enhancement ofetoricoxib by cosolvency approach. Int J Res 2012;86-93. https://doi.org/10.5402/2012/820653

3. Ruan LP, Yu BY, Fu GM, Zhu DN. Improving the solubility of ampelopsin by solid dispersions and inclusion complexes. J Pharma Biomed Res 2005;38:457-64.

4. Nighute $A B$, Bhise SB. Enhancement of dissolution rate of rifabutin by preparation of microcrystals using solvent changemethod. Int J PharmTech Res 2009;1:142-8.

5. Gupta PS, Sharma V, Pathak K. Melt sonocrystallized piroxicam for oral delivery: particle characterization, solidstate analysis and pharmacokinetics. Expert Opin Drug Delivery 2013;10:17-32.

6. Kumar B, Sharma V, Pathak K. Effect of meltsonocrystallization on pharmacotechnical properties of paracetamol, indomethacin and mefenamic acid characterized by dynamic laser scattering and its impact on solubility. Drug Dev Ind Pharm 2013;39:687-95.

7. Cavallari C, Abertini B, Rodriguez MG, Rodriguez L. Improved dissolution behaviour of steam-granulated piroxicam. Eur J Pharm Biopharm 2002;54:65-73.

8. Nazzal S, Khan MA. Controlled release of a self-emulsifying formulation from tablet dosage form: Stability assessment and optimization of some processing parameters. Int J Pharm 2006;315:110-21.

9. Kapsi SG, Ayres JW. Processing factors in development of solid solution formulation of itraconazole for enhancement of drug dissolution and bioavailability. Int J Pharm 2001;229:193-203.

10. Cole ET. Liquid-filled and sealed hard gelatin capsule technologies. $1^{\text {st }}$ Edition. Modified Release Drug Delivery Technology; 2003. p. 177-90.

11. Cui J, Yu B, Zhao Y, Zhu W, Li H, Lou H. Enhancement of oral absorption of curcumin by self-microemulsifying drug delivery systems. Int J Pharm 2009;371:148-55.

12. Lachman L, Leibermen HA, Kanig JK. The theory and practice of Industrial Pharmacy; 2002. p. 36-102, 184, 293.

13. Aulton ME. The science of dosage form design. 3rd ed.; 2008. p. 244-58.

14. Spireas S, Sadu S, Grover R. In vitro release evaluation of hydrocortisone liquisolid tablets. JPPS 1998;87:867-72.

15. Spirea S. Liquisolid systems and methods of preparing same. United State Patent 6; 2002;23:339.

16. Ali Nokhodchi, Christina M Hentzschel, Claudia S Leopold. Drug release from liquisolid systems: speed it up, slow it down. Expert Opin Drug Delivery 2011;8:191-205.

17. Banker GS, Anderson NL. Tablets. In: The theory and practice of industrial pharmacy. $3^{\text {rd }}$ ed. Lachman L, Liberman HA, Kanig JL; 1987. p. 293-345.

18. Fahmy RH, Kassem MA. Enhancement of famotidine dissolution rate through liquisolid tablet formulation: in vitro and in vivo evaluation. Eur J Pharm Biopharm 2008;69:993-1003.

19. Charman SA, Charman WN. Oral modified release delivery systems. In: Rathbone MJ, Hadgraftb J, Roberts MS. Modified Release Drug Delivery Technology; 2003. p. 1-9.

20. Yadav VB, Nighute AB, Yadav AV, Bhise SB. Aceclofenac size enlargement by non-aqueous granulation with improved solubility and dissolution. APSR; 2009. p. 115-22.

21. Spireas S, Sadu S. Enhancement of prednisolone dissolution properties using liquisolidcompacts. Int J Pharm 1998;166:177-88.
22. Spireas Sanford, Bolton. Liquisolid systems and methods of preparing same, United States Patent, US 5800834; 1998.

23. Spiridon, Spieras. Liquisolid systems and methods of preparing same. United States Patent US 6423339B1; 2012.

24. Elkordy AA, Tan XN, Essa EA. Spironolactone release fromliquisolid formulations prepared with Capryol $^{\text {TM }} 90$, Solutol@HS-15 and Kollicoat $®$ SR 30 D as non-volatile liquid vehicles. Eur J Pharm Biopharm 2013;83:203-23.

25. Saeedi M, Akbari J, Morteza Semnani K. Enhancement of dissolution rate of indomethacin using liquisolidcompacts. Iran J Pharm Res 2011;10:25-34.

26. Hentzschel CM, Sakmann A, Leopold CS. Suitability of various excipients as carrier and coating materials for liquisolid compacts. Drug Dev Ind Pharm 2011;37:1200-7.

27. Javadzadeh Y, Shariati H, Movahhed Danesh E. Effectof some commercial grades of microcrystalline cellulose on flowability, compressibility, and dissolution profile of piroxicam liquisolid compacts. Drug Dev Ind Pharm 2009;35:243-51.

28. Gavali SM, Pacharane SS, Sankpal SV. Liquisolid compact: a new technique for enhancement of drug dissolution. IJRPC 2011;1:705-13.

29. Akash S Bhise, Sapana Ahirrao, Amol D Rahane, Sanjay Kshirsagar. Solubility enhancement of poorly water-soluble drugs using liquisolid technique. IJOD 2018;6:152-64.

30. Tiong N, Elkordy. Effects of liquisolid formulations on the dissolution of naproxen. Eur J Pharm Biopharm 2009;73:373-84.

31. Wankhede Navneet B, Walekar SS, Sadgir PS, Pawar SA, Ahirrao SP. Liquisolid: a novel technique for dissolution enhancement of poorly water-soluble drugs. AJPTI 2014;2:77-90.

32. Onoue S, Yamada S, Chan HK. Nanodrugs: pharmacokineticsand safety. Int J Nanomed 2014;9:1025-37.

33. R Santosh Kumar, Jampana Divija Sai. Liquisolid compacts: a review. JDDT 2019;9:880-3.

34. Vijay Sharma, Kamla Pathak. Attempts and outcomes of liquisolid technology: an updated chronological compilation of innovative ideas and adjuvants in the field J. Pharm Biomed Res 2016;2:1-21.

35. Enugula Pavani, Sheik Noman, Izhar Ahmed Syed. Liquisolid technique-based sustained release tablet of trimetazidine dihydrochloride. Drug Invent Tuday 2013;5:302-10.

36. Naveen Chella, Nataraj Narra, Tadikonda Rama Rao. Preparation and characterization of liquisolid compacts for improved dissolution of telmisartan. J Drug Delivery 2014. https://doi.org/10.1155/2014/692793

37. Vinod Valjibhai Siju, Moinuddin Soniwala, Swati Nagar. A novel technique to enhance dissolution rate of cilnidipine using liquisolid compact and wet granulation. IJPSR 2017;9:160-8.

38. Akash S. Bhise, Sapana Ahirrao, Amol D Rahane, Sanjay Kshirsagar. Solubility enhancement of poorly water soluble drugs using liquisolid technique. IJOD 2018;6:152-64.

39. Puppala Raman Kumar, Puvvada Chaitanya, Rachakonda Kalyani, Rangisetti Mahalakshmi Poojitha, Udaru Bhavani, Siddela Tirumala Rao. Formulation and evaluation of curcumin liquisolid tablets. JPI 2019;8:368-74.

40. Matthew Lam, Taravat Ghafourian, Ali Nokhodchi. Liquisolid system and liqui mass systems are not the same. AAPS PharmSciTech 2020;21:105.

41. Javadzadeh Y, Musaajrezaei L, Nokhodchi A. Liquisolid technique as a new approach to suatain propranolol hydrochloride release from tablet matrices. Int J Pharm 2008;362:102-8.

42. De Espindola B, Reilly Beringhs Anderee O, Divya Songelia. Liquisolid pellets: a pharmaceutical technology strategy to improve the dissolution rate of ritonavir. Saudi Pharm J 2019;27:702-12.

43. Lam M, Ghafourian T, Nokhodchi A. Liqui-pellet: the emerging next-generation oral dosage form which stems from liquisolidconcept in combination with pelletization technology. AAPS PharmSciTech 2019;20:231.

44. Pezzini BR, Litha Thomas, Anjali Anil. Liquisolid technology applied to pellets: evaluation of the feasibility and dissolution performance using felodipine as a model drug. Chem Eng Res Des 2016;110:62-9. 